\section{Oral Cancer Detection Novel Strategies and Clinical Impact}

Editor: Prashanth Panta

Publisher: Springer International Publishing,

Switzerland

Language: English

ISBN: 978-3-319-61255-3

Edition: 1/e

Published: 2019

Pages: 314, illustrated

Price: $€ 118.99$

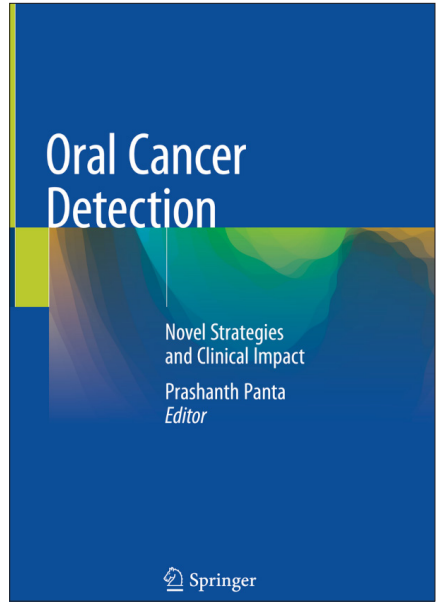

e-mail:

dr.vladimir.constantinescu@gmail.com
Despite the researchers' efforts, worldwide, more patients are diagnosed having cancer, and especially oral cancer. The book entitled "Oral Cancer Detection - Novel Strategies and Clinical Impact", coordinated by Dr. Prashanth Panta addresses all topics related to oral cancer, from precancerous lesions, epidemiology, imaging to histopathology. The book is divided into 15 chapters. The first chapters present general data on oral cancer, such as genetics and molecular mechanisms in oral cancer progression, respectively data on oral cancer epidemiology. After presenting standardized data for delayed diagnosis in oral cancer, the imaging methods used in oral cancer evaluation with the biopsy exam and histopathology in oral squamous cell carcinoma are also described. Assessment criteria for the clinical examination of the oral cavity are presented as the application of vital stains as adjuncts of clinical examination. Oral cancer investigations are consistently and convincingly described and illustrated by optical techniques, optical imaging, optical coherence tomography, bioimpedance, sensitive crystallization patterns, and salivary biomarkers. The book is clearly written, each chapter is abundantly illustrated, and is accompanied by up-to-date references on the subject.

Dr. Prashanth Panta, along with a team of clinicians, has been able to provide the reader with an excellent monograph on oral cancer detection. Oral cancer screening should be part of every initial and periodical examination of the oral cavity. Therefore, every clinician should consult this book. 